\title{
Measurement of ground-state decoherence via interruption of coherent population trapping
}

\author{
Anil K. Patnaik, ${ }^{1,2}$ Paul S. Hsu, ${ }^{1}$ Girish S. Agarwal, ${ }^{3}$ George R. Welch, ${ }^{1}$ and Marlan O. Scully ${ }^{1,2,4}$ \\ ${ }^{1}$ Institute for Quantum Studies and Department of Physics, Texas A\&M University, Texas 77843, USA \\ ${ }^{2}$ Applied Physics and Materials Science Group, Eng. Quad., Princeton University, New Jersey 08544, USA \\ ${ }^{3}$ Department of Physics, Oklahoma State University, Stillwater, Oklahoma 74078, USA \\ ${ }^{4}$ Max-Planck-Institute für Quantenoptik, Hans-Kopfermann-Strasse 1, D-85748, Garching, Germany \\ (Received 11 August 2006; revised manuscript received 31 October 2006; published 6 February 2007)
}

\begin{abstract}
We examine the role of ground-state coherence on the fluorescence from the excited state in a three-level $\Lambda$ system under continuous-wave excitation. When resonantly pumped, the fluorescence is strongly affected by any perturbation in the ground-state coherent population trapping. From our analytical results, we suggest how to use this strong dependence of fluorescence on the ground-state decays as a new method for the measurement of the ground-state decoherence rates. Our calculations should work well for a wide range of systems such as atomic vapors with a buffer gas, active dopants in solids, and quantum dots, etc. We present a proof of principle experiment using ${ }^{87} \mathrm{Rb}$ vapor.
\end{abstract}

DOI: $10.1103 /$ PhysRevA.75.023807

PACS number(s): 42.50.Gy, 42.50.Ct

\section{INTRODUCTION}

A popular method for determining the dephasing of the ground states is via Raman scattering because the linewidth of the scattering process is determined by the effective decay of the coherence between the initial and final states [1]. Similar information can be obtained from coherent anti-Stokes Raman scattering (CARS) [2] because the CARS line shape is related to the Raman line shape $[3,4]$. All this typically assumes pump and probe fields so that the resulting physical process is described by the third-order susceptibility. Compared to conventional Raman scattering, CARS is capable of high spectral resolution. However, it is limited by a stringent phase matching requirement. In what follows, we show how coherent population trapping (CPT) [5] can be used for the determination of the dephasing rates.

Further, the method we propose also enables one to determine the nonradiative rates that might come about from thermally stimulated processes [6]. As is well known, a dark state forms when no decay processes other than radiative decay are considered [7]. However, the dark state starts becoming bright due to nonradiative processes. Clearly the interruption of the dark state could be a sensitive method of studying nonradiative processes. This is reminiscent of studies in interferometry where the interferometer is tuned to a dark fringe. It has been observed in an earlier study that CPT is also very sensitive to internal parameters of the atom such as spontaneous emission linewidths [8].

The outline of this paper is as follows: we describe the system and derive dynamical equations in Sec. II. In Sec. III, we present numerical results to show how the excited-state population is affected due to interruption of CPT by groundstate decoherences. We also derive an analytical formula to understand the numerical result, and also to demonstrate the possibility of measuring various ground-state decoherence rates. In Sec. IV, we present the results of a proof-ofprinciple experiment using a ${ }^{87} \mathrm{Rb}$ cell with results in good agreement with the theory. Finally, we summarize and conclude in Sec. V. At the end, we present an appendix on the open system consideration and discuss the effect of time of flight of atom through the interaction region.

\section{THE SYSTEM AND THE DYNAMICS}

We consider a three-level $\Lambda$ system having all various possible decays such as coherence dephasing $\gamma_{b c}$ and nonradiative decays $\Gamma_{b}$ and $\Gamma_{c}$. The source of coherence dephasing can be due to collisions in gas cells [9] or phonon induced in solids [10], and the nonradiative decays could be incoherent pumpings between the ground states via thermal excitations in gas cells (particularly when the ground states are degenerate or near degenerate) or crystal-field-induced decays [6]. Two lasers couple the two ground states with the excited state. The pump (drive) laser couples the $a \leftrightarrow c(a \leftrightarrow b)$ transition with a Rabi frequency $2 \Omega_{p}=\wp_{a c} E_{p} / \hbar$ $\left(2 \Omega_{d}=\wp_{a b} E_{d} / \hbar\right)$. Here $\wp_{i j}=\langle i|\wp \wp| j\rangle$ represents the dipole moment corresponding to the $|i\rangle \leftrightarrow|j\rangle$ transition. In the following we briefly outline the derivation of the equation of dynamics.

The interaction Hamiltonian in rotating wave approximation (RWA) can be written as [7]

$$
\mathcal{H}_{I}=-\hbar \Omega\left[r|a\rangle\left\langle c\left|e^{-i \omega_{p} t}+\right| a\right\rangle\langle b| e^{-i \omega_{d} t}+\text { H.c. }\right],
$$

where $\omega_{p}\left(\omega_{d}\right)$ represents the central frequency pump (drive) field. We use the notation $r=\Omega_{p} / \Omega_{d}$ and $\Omega_{d}=\Omega$. The unperturbed Hamiltonian for this system (shown in Fig. 1) can be

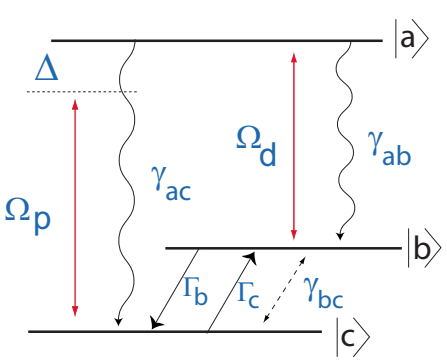

FIG. 1. (Color online) A three-level $\lambda$ system with ground-state coherence dephasing $\gamma_{b c}$ and also nonradiative decays $\Gamma_{b}$ and $\Gamma_{c}$ in the ground state. The spontaneous decay from $a \leftrightarrow b(a \leftrightarrow c)$ is given by $\gamma_{a b}\left(\gamma_{a c}\right)$. The pump field $\Omega_{p}$ is detuned by $\Delta$ from the $a \leftrightarrow c$ transition and the drive field $\Omega_{d}$ is on resonance with the $a \leftrightarrow b$ transition. 

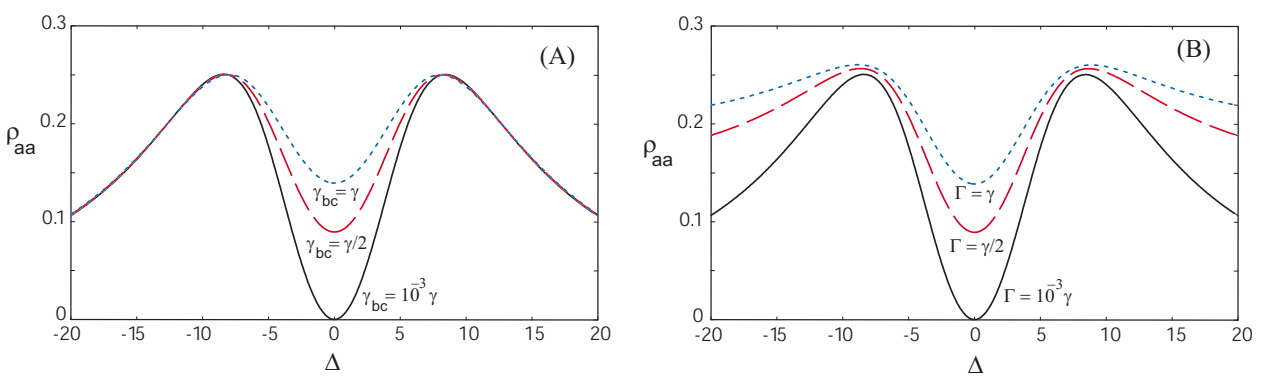

FIG. 2. (Color online) The excited population for various values of ground-state dephasing $\gamma_{b c}$ and nonradiative decays $\Gamma_{b}$ and $\Gamma_{c}$. The dependence of two decays are illustrated in (a) $\Gamma_{b}=\Gamma_{c}=\Gamma=0$ and (b) $\gamma_{b c}=0$. In all the plots, we have assumed $\gamma_{a b}=\gamma_{a c}=\gamma$, the laser Rabi frequency $\Omega=5 \gamma$ and $r=1$. written as

$$
\mathcal{H}_{0}=\hbar \omega_{a c}|a\rangle\left\langle a\left|+\hbar \omega_{b c}\right| b\right\rangle\langle b|,
$$

where $\hbar \omega_{i j}$ is the energy separation between the states $|i\rangle \leftrightarrow|j\rangle$. The equation of motion for the lambda system is given by the density-matrix equation

$$
\frac{\partial \rho}{\partial t}=-\frac{i}{\hbar}\left[\mathcal{H}_{0}+\mathcal{H}_{I}, \rho\right]+\text { decay terms }
$$

Next we make the following transformation $\rho \rightarrow \widetilde{\rho}$ to eliminate the rapid temporal oscillations

$$
\begin{gathered}
\rho_{a b} \rightarrow \tilde{\rho}_{a b} \exp \left[-i \omega_{d} t\right], \\
\rho_{a c} \rightarrow \tilde{\rho}_{a c} \exp \left[-i \omega_{p} t\right], \\
\rho_{b c} \rightarrow \widetilde{\rho}_{b c} \exp \left[-i\left(\omega_{p}-\omega_{d}\right) t\right], \\
\rho_{i i} \rightarrow \tilde{\rho}_{i i} .
\end{gathered}
$$

Thus the equations for the density-matrix elements with natural decay, ground-state dephasing, and nonradiative decay rates are

$$
\begin{gathered}
\frac{\partial \rho_{a a}}{\partial t}=-2\left(\gamma_{a b}+\gamma_{a c}\right) \rho_{a a}+i \Omega \rho_{b a}-i \Omega^{*} \rho_{a b}+i r \Omega \rho_{c a} \\
-i r^{*} \Omega^{*} \rho_{a c}, \\
\frac{\partial \rho_{a b}}{\partial t}=-\left(\gamma_{a b}+\gamma_{a c}+\Gamma_{b} / 2\right) \rho_{a b}-i \Omega\left(\rho_{a a}-\rho_{b b}\right)+i r \Omega \rho_{c b}, \\
\frac{\partial \rho_{a c}}{\partial t}=-\left(\gamma_{a b}+\gamma_{a c}+\Gamma_{c} / 2+i \Delta\right) \rho_{a c}+i \Omega \rho_{b c}-i r \Omega\left(\rho_{a a}-\rho_{c c}\right), \\
\frac{\partial \rho_{b b}}{\partial t}=-\Gamma_{b} \rho_{b b}+\Gamma_{c} \rho_{c c}+2 \gamma_{a b} \rho_{a a}-i \Omega \rho_{b a}+i \Omega^{*} \rho_{a b}, \\
\frac{\partial \rho_{b c}}{\partial t}=-\left[\gamma_{b c}+\left(\Gamma_{b}+\Gamma_{c}\right) / 2+i \Delta\right] \rho_{b c}+i \Omega^{*} \rho_{a c}-i r \Omega \rho_{b a}, \\
\frac{\partial \rho_{c c}}{\partial t}=-\Gamma_{c} \rho_{c c}+\Gamma_{b} \rho_{b b}+2 \gamma_{a c} \rho_{a a}-i r \Omega \rho_{c a}+i r^{*} \Omega^{*} \rho_{a c} .
\end{gathered}
$$

Here $\Gamma_{b}\left(\Gamma_{c}\right)$ is the nonradiative decay from state $b$ to $c(c$ to $b), 2 \gamma_{i j}$ are the decay rates from $|i\rangle \rightarrow|j\rangle, \gamma_{b c}$ is the dephasing rate of the Raman coherence, and $\Delta$ is the pump field detuning (see Fig. 1). In the following section we solve the above dynamic equation to determine how fluorescence is affected by all these decay rates.

\section{RESULTS AND DISCUSSIONS}

We have numerically solved Eqs. (5) and plotted the steady-state excited state population $\rho_{a a}$ in Fig. 2. This depicts how the fluorescence from the atom depends on pump field detuning. We see that when both fields $\Omega_{b}$ and $\Omega_{c}$ are on resonance, the population reaches a minimum for a zero dephasing rate $\gamma_{b c}$ and nonradiative decay rates $\Gamma_{b}$ and $\Gamma_{c}$. This is clearly due to CPT. However, for finite $\gamma_{b c}$ and $\Gamma_{b}$ $=\Gamma_{c}=\Gamma$, the CPT is perturbed and hence population from the dark state (antisymmetric superposition of ground state) partially transfers to the bright state (symmetric superposition of the ground states). Thus the excited state gets populated by its coupling to the bright state and hence starts fluorescing. In what follows below, we will show how this significant variation in the fluorescence signal at resonance could be used to measure the ground-state decoherence rates.

To isolate the roles of ground state dephasing and nonradiative decay we have plotted $\rho_{a a}$ in Fig. 2(a) assuming the nonradiative decay $\Gamma_{b}=\Gamma_{c}=0$, and in Fig. 2(b) we have shown the excited-state population when only nonradiative decay is present but $\Gamma_{b c}=0$. Clearly, $\gamma_{b c}$ affects the fluorescence only around the resonance as it only disturbs the coherence created in the ground state. As a result, some population is made available at the bright state to couple to the excited state via the laser field and hence start fluorescing. Clearly, the larger $\gamma_{b c}$ is, the stronger the fluorescence at $\Delta$ $=0$. However, the nonradiative decays affect the fluorescence profile even for the wide off-resonant field because $\Gamma_{b}$ and $\Gamma_{c}$ incoherently mix populations in the ground state in addition to causing ground-state decoherence.

To understand the above argument, we have calculated $\rho_{a a}$ analytically for both resonant and off-resonant cases in the limit of intense lasers. In the following we present the solutions with nonradiative decay between ground states included in the calculation, and assuming that $\Omega$ $\gg \gamma_{a b}, \gamma_{a c}, \gamma_{b c}, \Gamma_{b}, \Gamma_{c}$. For $r=1$ and $\Gamma_{b}=\Gamma_{b}=\Gamma, \gamma_{a b}=\gamma_{a c}=\gamma$, the steady-state solution of Eq. (5) for the off-resonant pump laser $(\Delta \neq 0)$ is obtained as 


$$
\rho_{a a}=\frac{A_{1}\left(4 \Omega^{4} A_{2}+\Delta^{4} \Gamma\right)+\Delta^{2} \Omega^{2}\left\{4 \gamma A_{1}+2 \Gamma\left(2 \gamma+\gamma_{b c}\right)+3 \Gamma^{2}\right\}}{A_{1}\left\{4 \Omega^{4}\left(3 A_{2}+4 \gamma\right)+\Delta^{4} A_{3}\right\}+\Delta^{2} \Omega^{2}\left\{2 A_{3}\left(4 \gamma+A_{2}\right)+3 \Gamma^{2}\right\}},
$$

where

$$
A_{1}=4 \gamma+\Gamma, \quad A_{2}=\Gamma+\gamma_{b c}, \quad \text { and } \quad A_{3}=2 \gamma+3 \Gamma .
$$

This analytical result agrees very well with the numerical result presented above. Clearly, in Fig. 2(a), the value of $\rho_{a a}$ at resonance (i.e., $\Delta=0)$ is given by $\rho_{a a} \equiv \gamma_{b c} /\left(3 \gamma_{b c}+4 \gamma\right)$. For $\gamma_{b c} \ll \gamma$, the excited-state population under the condition of CPT depends linearly on $\gamma_{b c}$, i.e., $\rho_{a a} \approx \gamma_{b c} /(4 \gamma)$. Similarly, in Fig. 2(b), $\rho_{a a}$ at $\Delta=0$ is given by $\rho_{a a}=\left(\Gamma_{b}+\Gamma_{c}\right) /\left[3\left(\Gamma_{b}+\Gamma_{c}\right)+8 \gamma\right]$.

Further, we get a more general result for the resonant pumping. Again, we have considered strong pump and drive lasers but $r \neq 1\left(\Omega_{p} \neq \Omega_{d}\right)$. We could even keep unequal nonradiative decays between the ground states $\Gamma_{b} \neq \Gamma_{d}$. The solution for this case is obtained as

$$
\rho_{a a}=\frac{\Gamma_{b} r^{4}+2 \gamma_{b c} r^{2}+\Gamma_{c}}{\Gamma_{b}\left(1+2 r^{4}\right)+\Gamma_{c}\left(2+r^{4}\right)+6 \gamma_{b c} r^{2}+2 \gamma\left(1+r^{2}\right)^{2}} .
$$

Assuming there is no coherence dephasing in the system (i.e., $\gamma_{b c}=0$ ), the above expression reduces to that obtained in Ref. [4]. For $r=1$, the above equation further simplifies to

$$
\rho_{a a}=\frac{2 \gamma_{b c}+\Gamma_{b}+\Gamma_{c}}{3\left(2 \gamma_{b c}+\Gamma_{b}+\Gamma_{c}\right)+8 \gamma} .
$$

This matches exactly with the numerical results presented above. Next, in Fig. 3 we show a numerical plot of how $\rho_{a a}$ varies with the change in ratios of the intensities $r^{2}$, when both the fields are on resonance. These plots match perfectly with the above analytical result. It should be noted that these

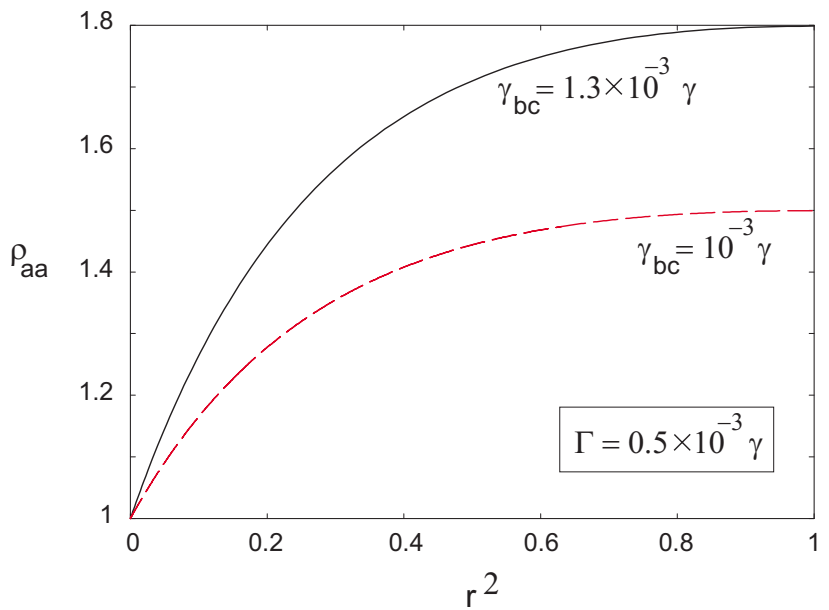

FIG. 3. (Color online) Plot of normalized $\rho_{a a}$ with respect to their values at $r=0$ vs the intensity ratio $r^{2}$. Here $\gamma_{a b}=\gamma a c=\gamma$ and $\Omega=5 \gamma$. Both pump and drive fields are on resonance here. analytical results also hold well for $r=0$ as long as $\Omega$ is assumed to be strong.

Equation (8) is very important, as it can be written as a linear equation in terms of the decoherence rates $\gamma_{b c}, \Gamma_{b}$, and $\Gamma_{c}$, given by

$$
\kappa_{1} \Gamma_{b}+\kappa_{2} \Gamma_{c}+\kappa_{3} \gamma_{b c}+\kappa_{0}=0
$$

where

$$
\begin{gathered}
\kappa_{1}=\left(1+2 r^{4}\right) \rho_{a a}-r^{4}, \quad \kappa_{2}=\left(2+r^{4}\right) \rho_{a a}-1, \\
\kappa_{3}=2 r^{2}\left(3 \rho_{a a}-1\right), \quad \text { and } \quad \kappa_{0}=2 \gamma\left(1+r^{2}\right)^{2} \rho_{a a} .
\end{gathered}
$$

The coefficients $\kappa_{i}$ are functions of measurable quantities such as the ratio of intensities $\left(r^{2}\right)$ of the two applied fields and the excited-state population, which is proportional to the fluorescence intensity. In an experiment, that would mean we need three values of $\rho_{a a}$ for different $r^{2}$ to determine all three ground-state decoherence rates. In this spirit, we can observe in Fig. 3 that indeed $\rho_{a a}$ depends very strongly on $\gamma_{b c}$ and $\Gamma_{i}$ $(i=b, c)$. In the plot we have normalized the $\rho_{a a}$ with their corresponding values at $r=0$.

It may be noted that this method should be valid for varieties of systems having the generic model of a three-level lambda scheme. Some possible systems of interest could be atoms in a buffer gas [11], molecules doped in solid [12-14], or multilevel quantum dots [15].

\section{EXPERIMENT}

In this section we present the results of a proof-ofprinciple experiment that agrees qualitatively with the above discussions. At the end of this section we discuss in detail some limits in implementing the exact idea in ${ }^{87} \mathrm{Rb}$ gas.

The experimental setup is shown schematically in Fig. 4. An external cavity diode laser (ECDL) is tuned to the $5 S_{1 / 2}(F=2) \rightarrow 5 P_{1 / 2}(F=1)$ transition of the $\mathrm{Rb} D_{1}$ line. The laser linewidth is about $100 \mathrm{kHz}$. The laser beam is split into two by a 50/50 beam splitter, the polarization of one of the beams is rotated by a $\lambda / 2$ wave plate, and these two orthogonal linearly polarized beams are then combined with a polarizing beam splitter (PBS). After the combined beam passes through the $\lambda / 4$ wave plate, the beam is a combination of two orthogonal circularly polarized components. These components couple two Zeeman ground-state sublevels to the single excited state as shown in Fig. 4(b). Thus compared to the model in Fig. 1, the right-circular polarization component would correspond to $\Omega_{p}$ and the left-circular polarization component would correspond to $\Omega_{d}$. The intensity for the left- and right-circular polarization components can be attenuated by placing polarizers after the $\lambda / 2$ wave plate. The laser beam diameter can be controlled with a telescope beam expander. The beam then passes through a cylindrical glass 


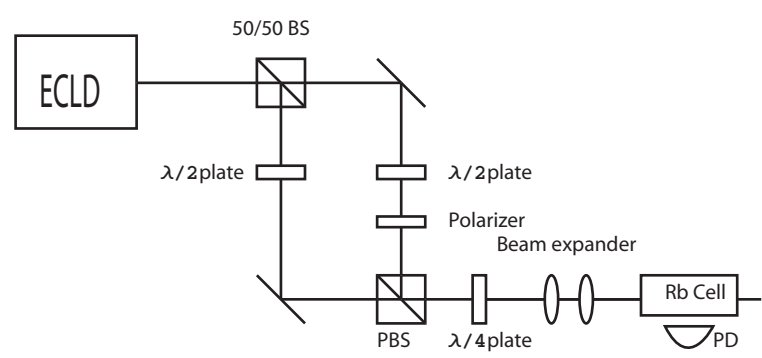

(a)

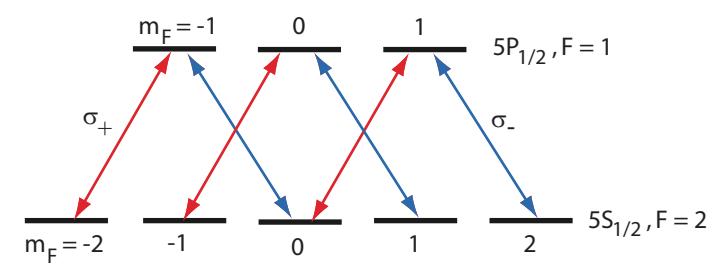

(b)

FIG. 4. (Color online) (a) The experimental setup to detect the fluorescence as a function of intensity ratio $r^{2}$. (b) The hyperfine Zeeman sublevel transitions of ${ }^{87} \mathrm{Rb}$ under consideration.

cell of length $10 \mathrm{~cm}$ containing rubidium vapor. At room temperature, the $\mathrm{Rb}$ density is approximately $8 \times 10^{9} \mathrm{~cm}^{-3}$.

The laser power in each beam at the entrance of the $\mathrm{Rb}$ cell is approximately $3 \mathrm{~mW}$. The fluorescence signal is registered with an amplified Si detector (PDA55) with $10 \mathrm{MHz}$ frequency bandwidth. Fluorescence is detected at the side of the cell, perpendicular to the laser propagation direction. Care is taken to minimize residual scattered light from the glass wall of the cell. To compensate for this scattered light, the signal is measured when the laser is detuned far away from resonance so there is no fluorescence, and this background is subtracted from the fluorescence signal. In the experiment, we measure the fluorescence emission from the excited state for various ratios of intensities $r^{2}$. To minimize the experimental uncertainty, we fix the intensity of the leftcircular polarized component $\Omega_{d}$ as the reference beam and only varied the intensity for the right-circular component $\Omega_{p}$.

The different laser beam diameters $D$ give rise to different ground-state dephasing rates because the thermally moving atoms spend more time on the average in a larger beam. Coherence is lost when the atoms collide with the walls, and thus in a larger beam one would expect a lower rate of dephasing collisions. So the maximum coherence time (and so the minimum $\gamma_{b c}$ ) is determined by the laser beam diameter. We present here data sets for two different values of $D$. Since the total number of atoms contributing to the fluorescence changes with the beam diameter, we have normalized the fluorescence signals by their corresponding values at $r$ $=0$. Further, the number of atoms in the interaction region for a given beam diameter is the same for both $r=0$ and $r \neq 0$. Thus, the normalized fluorescence becomes independent of the number of atoms participating in the fluorescence. The results are shown in Fig. 5. These agree qualitatively with the behavior shown in Fig. 3 calculated from the three-level model.

Although the data agree qualitatively with the analytical calculations, we cannot derive accurate values for the

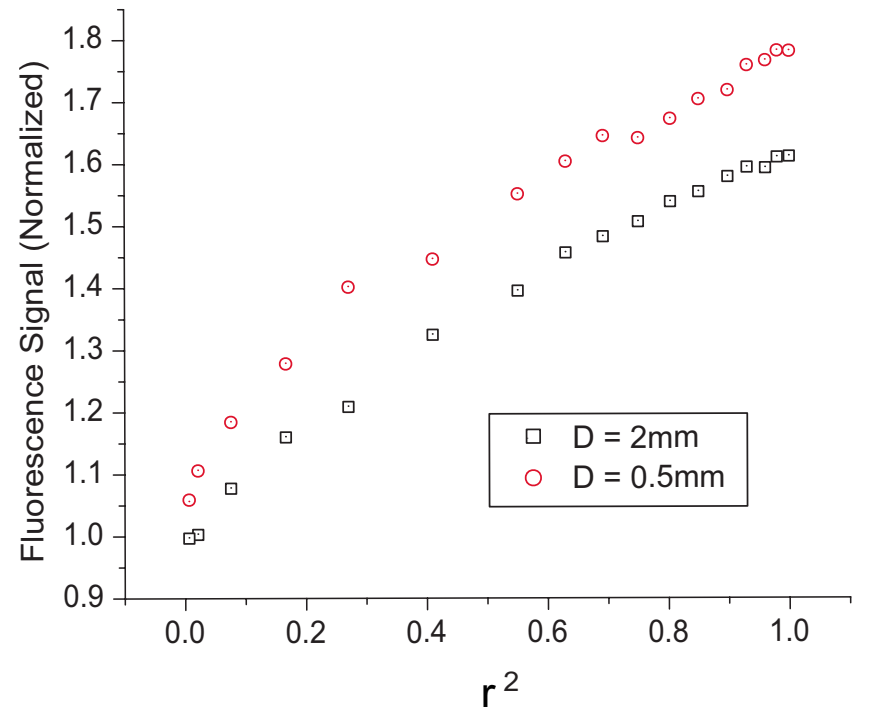

FIG. 5. (Color online) Experimental fluorescence data (normalized with their corresponding $r=0$ ) under CPT vs the intensity ratio $r^{2}$. A larger value for beam diameter $D$ would result in smaller $\gamma_{b c}$.

dephasing rates. To do so, we would need to extract $\rho_{a a}$ from the data, which would require calibrating the measured fluorescence to the total fluorescence from the atoms. One way of performing this calibration would be to incoherently pump the excited state and detect the fluorescence with a detector of known efficiency. However, such pumping in ${ }^{87} \mathrm{Rb}$ would also excite the other upper (or lower) hyperfine components. [The upper $5 P_{1 / 2}(F=2)$ state considered here is only $800 \mathrm{MHz}$ from the $5 P_{1 / 2}(F=1)$ state, and only partially resolved due to the inhomogeneous Doppler broadening of $500 \mathrm{MHz}$.] Therefore, our theory would be exact for systems having reasonably isolated ground and excited states.

It may also be noted that the model system for the theoretical calculation given in Sec. 3 corresponds to a closed system. However, the proof-of-principle experiment on the ${ }^{87} \mathrm{Rb}$ gas cell corresponds to an open system. It has been observed that the CPT resonance strongly depends on the strength of the driving field in an open system [16]. It is well known that the population loss in the cell due to finite time of flight of the gaseous atoms through the interaction region (laser beam) contributes as an additional effective groundstate dephasing rate [17], though the dynamics population itself is not affected as much (see the Appendix). The aforesaid underlying principle thus remains the same for both closed and open systems in the determination of the collisional dephasing rate, except that one has to account for the finite time of flight of gas atoms. The average time of flight can be determined as

$$
t_{\text {flight }}=D \sqrt{\frac{3 k_{B} T}{M_{a}}},
$$

where $D$ is the diameter of the laser beam, $T$ is the temperature of the cell, $M_{a}$ is the mass of the atom, and $k_{B}$ is the Boltzmann constant. Therefore, the actual value of the collisional dephasing rates in the gas cell would be given by 


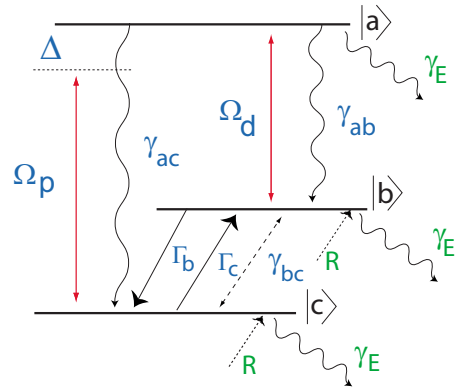

FIG. 6. (Color online) The model for an open system. Here $R$ is the rate at which atoms enter into the interaction region and $\gamma_{E}$ is the effective decay of the atomic population due to the exit of atoms from the interaction region.

subtracting $\left(t_{\text {flight }}\right)^{-1}$ from the experimentally measured value of ground-state dephasing.

\section{SUMMARY}

We have examined the role of ground-state decoherence rates on the fluorescence in a resonant three-level lambda system. We have shown that the fluorescence is strongly affected by the ground-state decoherence due to interruption of the coherent population trapping. We have theoretically demonstrated how to exploit this sensitivity to measure groundstate decoherence rates including the nonradiative decays via an analytical solution. We have presented a proof-ofprinciple experiment that is in conformity with our theoretical calculation. We have noted that this method should work well for a wide variety of systems.

\section{ACKNOWLEDGMENTS}

We are thankful to Professor E. Arimondo and Professor K. Hakuta for useful discussions on the implementation of this method, and to V. A. Sautenkov and Aihua Zhang for useful discussions and help on the experimental setup. We thank DARPA, ONR, and Robert A. Welch Foundation (Grant No. A-1261) for support.

\section{APPENDIX: OPEN SYSTEM MODEL TO DESCRIBE TIME-OF-FLIGHT EFFECT}

In this appendix we describe briefly the open system considerations for the system of our interest. The system to describe the effect of artificial decays due to finite time of interaction of the atoms during the time of flight through the laser beams could be modeled as shown in Fig. 6. Correspondingly, our equations of motion (5) will be modified to

$$
\begin{aligned}
\frac{\partial \rho_{a a}}{\partial t}= & -2\left(\gamma_{a b}+\gamma_{a c}+\gamma_{E}\right) \rho_{a a}+i \Omega \rho_{b a}-i \Omega^{*} \rho_{a b} \\
& +i r \Omega \rho_{c a}-i r^{*} \Omega^{*} \rho_{a c},
\end{aligned}
$$

$$
\begin{gathered}
\frac{\partial \rho_{a b}}{\partial t}=-\left(\gamma_{a b}+\gamma_{a c}+2 \gamma_{E}+\Gamma_{b} / 2\right) \rho_{a b} \\
-i \Omega\left(\rho_{a a}-\rho_{b b}\right)+i r \Omega \rho_{c b}, \\
\frac{\partial \rho_{a c}}{\partial t}=-\left(\gamma_{a b}+\gamma_{a c}+2 \gamma_{E}+\Gamma_{c} / 2+i \Delta\right) \rho_{a c}+i \Omega \rho_{b c} \\
-i r \Omega\left(\rho_{a a}-\rho_{c c}\right), \\
\frac{\partial \rho_{b b}}{\partial t}=R-2 \gamma_{E} \rho_{b b}-\Gamma_{b} \rho_{b b}+\Gamma_{c} \rho_{c c}+2 \gamma_{a b} \rho_{a a} \\
-i \Omega \rho_{b a}+i \Omega^{*} \rho_{a b}, \\
\frac{\partial \rho_{b c}}{\partial t}=-\left[2 \gamma_{E}+\gamma_{b c}+\left(\Gamma_{b}+\Gamma_{c}\right) / 2+i \Delta\right] \rho_{b c}+i \Omega^{*} \rho_{a c}-i r \Omega \rho_{b a} \\
\frac{\partial \rho_{c c}}{\partial t}=R-2 \gamma_{E} \rho_{c c}-\Gamma_{c} \rho_{c c}+\Gamma_{b} \rho_{b b}+2 \gamma_{a c} \rho_{a a}-i r \Omega \rho_{c a} \\
+i r^{*} \Omega^{*} \rho_{a c} .
\end{gathered}
$$

Here, $R$ is the rate at which atoms enter into the interaction region and $\gamma_{E}$ is the effective decay of the atomic population due to the exit of atoms from the interaction region given by $\left(t_{\text {flight }}\right)^{-1}$. Here $t_{\text {flight }}$ is the flight time of the atom through the laser beams. Demanding that in steady state the total change in poulation should be zero, i.e.,

$$
\frac{\partial \rho_{a a}}{\partial t}+\frac{\partial \rho_{b b}}{\partial t}+\frac{\partial \rho_{c c}}{\partial t}=0,
$$

gives

$$
\rho_{a a}+\rho_{b b}+\rho_{c c}=\frac{R}{\gamma_{E}}
$$

Thus to restrict any buildup of population in the interaction region, we need the condition $R=\gamma_{E}$. Now we make a very approximate estimate as in the following.

(1) Since the time of flight is an order of milliseconds as compared to the spontaneous decay time of an order of nanoseconds, we can drop $\gamma_{E}$ compared to $\gamma_{i j}$ in the equations for $\rho_{a a}, \rho_{a b}$, and $\rho_{a c}$.

(2) Moreover, as we are working under the CPT condition, if we assume $\rho_{b b} \approx \rho_{c c} \approx 0.5$ (and also using the condition that $R=\gamma_{E}$ ), the first two terms in both equations for $\rho_{b b}$ and $\rho_{c c}$ will cancel with each other.

Thus all the above equations in Eqs. (A1) reduce to exactly the same form as in the closed system Eqs. (5), except for the equation for ground-state coherence $\rho_{b c}$, because $\gamma_{E}$ is comparable to $\gamma_{b c}$. Therefore, in our calculation the timeof-flight-induced decay primarily affects the dephasing and the population decay is not affected as much. 
[1] W. Demtröder, Laser Spectroscopy (Springer-Verlag, New York, 2002).

[2] Y. R. Shen, Principles of Non-linear Optics (Wiley, New York, 1984).

[3] F. DeMartini and J. Ducuing, Phys. Rev. Lett. 17, 117 (1966); for a review see A. Laubereau and W. Kaiser, Rev. Mod. Phys. 50, 607 (1978).

[4] G. S. Agarwal and S. S. Jha, J. Phys. B 12, 2655 (1979).

[5] E. Arimondo, Prog. Opt. 35, 259 (1996).

[6] D. G. Steel and J. F. Lam, Phys. Rev. Lett. 43, 1588 (1979).

[7] M. O. Scully and M. S. Zubairy, Quantum Optics (Cambridge University Press, Cambridge, England 1997).

[8] I. V. Jyotsna and G. S. Agarwal, Phys. Rev. A 52, 3147 (1995).

[9] R. Loudon, Quantum Theory of Light (Oxford University Press, Cambridge, England 2001).

[10] J. Z. Li, M. Katsuragawa, M. Suzuki, and K. Hakuta, Phys. Rev. A 58, R58 (1998).

[11] E. E. Mikhailov, I. Novikova, Y. V. Rostovtsev, and G. R. Welch, Phys. Rev. A 70, 033806 (2004).
[12] For doped organic molecule, see B. Lounis and W. E. Moerner, Nature (London) 407, 491 (2000).

[13] For nitrogen vacancy in diamond, see C. Kurtsiefer, S. Mayer, P. Zarda, and H. Weinfurter, Phys. Rev. Lett. 85, 290 (2000); R. Brouri, A. Beveratos, J.-P. Poizat, and P. Grangier, Opt. Lett. 25, 1294 (2000).

[14] For a similar scheme in orthohydrogen doped in parahydrogen substrate, see A. K. Patnaik, J. Q. Liang, and K. Hakuta, Phys. Rev. A 66, 063808 (2002); J. Mod. Opt. 50, 2595 (2003).

[15] E. A. Muljarov, T. Takagahara, and R. Zimmermann, Phys. Rev. Lett. 95, 177405 (2005); P. Borri, W. Langbein, U. Woggon, M. Schwab, M. Bayer, S. Fafard, Z. Wasilewski, and P. Hawrylak, ibid. 91, 267401 (2003).

[16] F. Renzoni, W. Maichen, L. Windholz, and E. Arimondo, Phys. Rev. A 55, 3710 (1997).

[17] J. Vanier and C. Audoin, The Quantum Physics of Atomic Frequency Standards (Institute of Physics Publishing, Bristol, 1989); for a review on effect of time of flight, see W. Happer, Rev. Mod. Phys. 44, 169 (1972). 